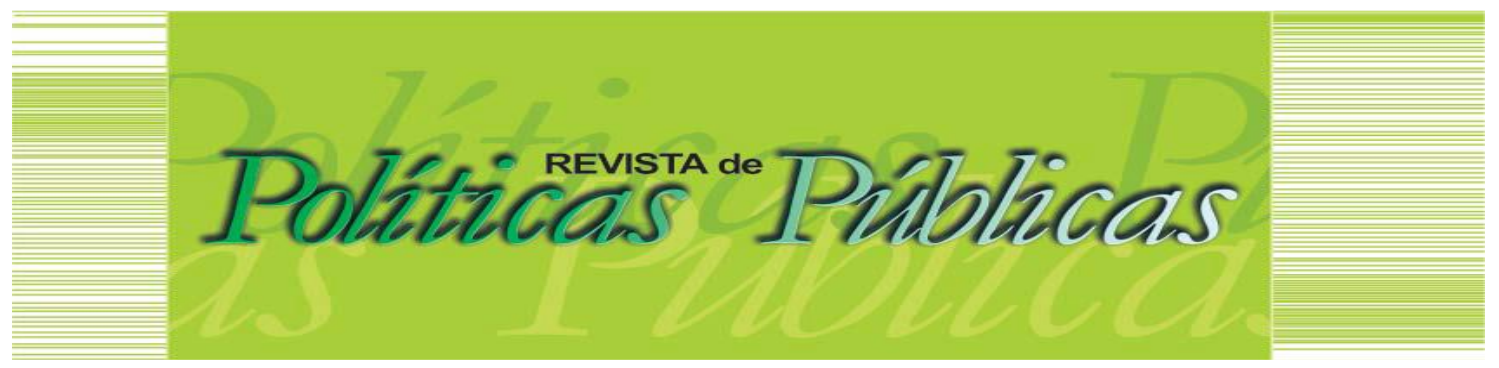

\title{
ARTICULAÇÃO E REDES NO ENFRENTAMENTO À VIOLÊNCIA CONTRA AS MULHERES NO PARÁ
}

\author{
Marcela Fernanda da Paz de Souza ${ }^{1}$ \\ Frederico Oliveira Henriques ${ }^{2}$
}

\begin{abstract}
Resumo:
O objetivo da pesquisa é verificar a formação de uma rede de enfrentamento à violência contra as mulheres do campo, das florestas e das águas no Estado do Pará. 0 estudo realiza o levantamento dos dados primários junto à CIPM-PA, e, baseandose na abordagem de rede social, construi a rede geral com os 39 atores da CIPM-PA, subdivida entre o Fórum Estadual de Enfrentamento à Violência Contra as Mulheres do Campo, das Florestas e das Águas, o Conselho Estadual de Mulheres e 0 Comitê de Monitoramento do Pacto Nacional de Violência Contra a Mulher. A análise da rede social observou o clique e as medidas de centralidade de informação, de grau, de proximidade, de intermediação. Assim, é possível concluir que o estudo da formação da rede de enfrentamento e da proeminência dos atores poderá contribuir com processos decisórios e estratégias de ação direcionadas às mulheres dos contextos rurais.
\end{abstract}

Palavras-chaves: Rede de Enfrentamento. Violência. Ruralidades

\section{ARTICULATION AND NETWORKS IN FACING VIOLENCE AGAINST WOMEN IN PARÁ}

\section{Abstract:}

The objective of this research is to verify the formation of a confrontation network against violence on women from the field, forests and waters in the state of Pará . The primary data survey was done joined with the CIPM -PA. Based on the social networking approach, the comprehensive network with 39 actors in the CIPM -PA was build up, subdivided between the State Forum to Fight Violence Against Field, Forestry and Water women, the State Council of Women and the Monitoring Committee of the National Pact of Violence Against Women. The analysis of the social network observed the click and the measures of centrality of information, degree, proximity, intermediation. Thus, it is possible to conclude that the study of the formation of the network of coping and of the prominence of the actors can contribute with decision-making processes and strategies of action directed to the women of the rural contexts..

Keywords: Coping Network. Violence. Ruralities

Artigo recebido em: 30/11/2018 Aprovado em: 04/04/2019. DOI: http://dx.doi.org/10.18764/2178-2865.v23n1p45-61

\footnotetext{
1 Bacharel em Comunicação Social. Doutora em Ciências Sociais pelo Programa de Pós-Graduação em Ciências Sociais (PPGCSO) pela Universidade Federal de Juiz de Fora (UFJF) com pós-doutorado em Estudos Urbanos e Regionais na Universidade Federal do Rio Grande do Norte (UFRN). Professora adjunta da Universidade do Estado de Minas Gerais (UEMG). Endereço: Av. Professor Mário Palmerio, 1001 - Bairro Universitário - Frutal/MG. CEP: 38200-000. E-mail: marcela.souza@uemg.br

2 Bacharel em Ciências Sociais. Doutorando no Programa de Ciências Sociais da UFRN. Diretor Técnico da Fundação Lauro Campos e Marielle Franco. Endereço: Al. Barão de Limeira, no 1400 - Campos Elíseos - São Paulo - SP. CEP 01202-002. Email: fredhenriqs@gmail.com
} 


\section{INTRODUÇÃO}

\section{Estudos (INSTITUTO BRASILEIRO DE GEOGRAFIA E ESTATÍSTICA, 2017; MATOS,} MEDEIROS, 2011; SUZIKI, 2007) atestam o aprofundamento da complexificação socioespacial entre campo e cidade. No que tange às transformações no campo, na análise de Durán (1998), há a inseparabilidade do rural e das ruralidades, cuja apreciação ocorre em uma abordagem de construção social, como produto da ação social dos sujeitos que se posicionam reflexivamente sobre as circunstâncias sociais e culturais, em suas temporalidades e espacialidades. Este espaço socionatural, parte da contínua construção da realidade social (SUÁREZ $R, 2008$ ), é tecido em suas funcionalidades pelos empreendimentos produtivos e de autossustentabilidade econômica, da utilização sustentável do território, do cuidado ecológico e ambiental, do investimento agroindustrial, somados às ações de recreações e lazer, entre outras esferas. Na explicação de Medeiros (2017, p. 183), os olhares sobre esse Brasil rural contemplam ruralidades, cujas análises abrangem os seguintes elementos inter-relacionados: "[...] ao espaço físico (referência ao território e aos seus símbolos), ao lugar onde se vive (territorialidades, identidades) e lugar de onde se vê e se vive o mundo (a cidadania e inserção nas esferas políticas e econômicas da sociedade)".

Essas indicações de leituras sobre o novo rural e as ruralidades, ao considerar, especialmente, a teia social constitutiva, direcionam para ampliar a compreensão da força que os movimentos de combate à violência podem assumir se as relações tecidas e reconhecidas nessas novas análises de ruralidades se propuserem à formação das redes de enfrentamento. A abrangência das mulheres da mata e das florestas adotada neste estudo compreende "[...] as mulheres trabalhadoras rurais, mulheres que vivem no campo, na ruralidade e na floresta, agricultoras familiares, as extrativistas, catadoras de côco e babaçu e as seringueiras." (BRASIL, 2011b, p. 10). A expressão mulheres da água se refere àquelas que vivem em regiões ribeirinhas, como na Região de Integração de Tapajós e na llha de Marajó, no Estado do Pará.

A violência contra as mulheres do campo e das florestas permaneceu, por muito tempo, negligenciada na ação pública e nas pesquisas, o que implica uma lacuna na compreensão dos processos, dos significados e das consequências da violência doméstica e familiar nas múltiplas ruralidades (BARROSO, 2011; BARSTED, 2006a, 2006b; 2011; DARON, 2009). É nesse cenário que os movimentos para as mulheres do campo e das florestas têm atuado decisivamente no enfrentamento à violência e na proposição de pautas na agenda governamental. São eles os principais atores dos movimentos que 


\section{ARTICULAÇÃO E REDES NO ENFRENTAMENTO À VIOLÊNCIA CONTRA AS MULHERES NO PARÁ}

emergiram da luta dos vários grupos femininos no campo. É em resposta às demandas e às reivindicações que houve a elaboração das ações de enfrentamento à violência contra as mulheres do campo e das florestas. (BRASIL, 2011b).

A rede de enfrentamento à violência contra a mulher deve ser compreendida como um esforço de articulação entre os diversos setores responsáveis pela gestão e execução das políticas transversais direcionadas às mulheres em situação de violência. Sua constituição inclui atores públicos e privados, formais e informais, responsáveis pelo planejamento, execução e avaliação dessas políticas; pelos serviços e programas de responsabilização dos agressores e pelo atendimento às vítimas; pelas secretarias federais, estaduais e municipais e pelas universidades. Essa articulação deve ser projetada de forma estratégica para que as vítimas sejam atendidas no combate, prevenção, assistência e garantia de direitos. (BRASIL, 2011a, 2011b). 0 conceito de rede se coaduna com a dimensão de enfretamento. Este implica na execução de políticas públicas articuladas, abrangentes, com capacidade para corresponder às demandas complexas e particulares dos diversos contextos socioeconômicos, territoriais e culturais destas mulheres em situação de violência. (BRASIL, 2011a, 2011b).

O enfretamento prescinde ações em rede, intersetoriais, que abranjam elementos da saúde, da assistência social e jurídica, da segurança pública, da habitação. São necessárias ações de caráter convergentes e transversais que possibilitem o cuidado integral da criança, da adolescente e da mulher vítima ou em situação de violência (BRASIL, 2011a, 2011b).

Ações de Enfrentamento à Violência contra as Mulheres do Campo e das Florestas

Espera-se, com a pesquisa, verificar se há a formação de uma rede de enfrentamento à violência familiar e doméstica contra as mulheres da mata, das florestas e das águas viabilizada pela Secretaria de Estado de Justiça e Direitos Humanos (SEJUDH)/Coordenadoria de Promoção de Políticas para as Mulheres (CPPM-PA). A hipótese com a qual trabalhamos é que em razão da CIPM apresentar a função de estimular a criação de organismos municipais, de viabilizar as parcerias com os conselhos, de articular a implementação das políticas que abranjam a diversidade das mulheres do campo, das florestas e das águas no Pará (BRASIL, 2014), ela pode fortalecer a ação de uma rede capaz de promover e interiorizar o acesso das mulheres vítimas de violência às redes de enfrentamento. 


\section{NOTAS METODOLÓGICAS}

Para cumprir esse objetivo, junto à revisão bibliográfica foi realizado o levantamento dos dados primários junto à Coordenadoria de Promoção de Políticas para as Mulheres do Estado do Pará por meio da aplicação do questionário Política para as Mulheres e Diversidade - Diversidade da Mulher e Mulheres do Campo, das Florestas e das Águas. Somou-se a essa etapa da pesquisa, o levantamento dos dados secundários através dos portais do Governo do Estado do Pará, da Secretaria de Política para as Mulheres da Secretaria da República.

A SEJUDH/CPPM-PA atua no âmbito da Coordenadoria Integrada de Políticas para as Mulheres (CIPM-PA), subdivida no Fórum Estadual de Enfrentamento à Violência das Mulheres do Campo, das Florestas e das Águas, no Comitê de Monitoramento do Pacto Nacional de Violência Contra a Mulher e no Conselho Estadual de Mulheres, com um total de 39 atores.

A pesquisa delimitou, para a análise da constituição da rede de enfrentamento, as relações estabelecidas entre esses atores na CIPM-PA. Através das medidas de centralidade é possível perceber a influência e o poder de determinado ator na rede (MARTELETO, 2001; WASSERMAN; FAUST, 1999).

A metodologia das redes sociais é um "[...] método para a descrição e para a análise dos padrões de relações" (MARQUES, 1998, p. 15) presentes nas entidades sociais. Adota-se, neste estudo, a abordagem de redes com ênfase nas redes sociais, abrangendo a linha de aplicabilidade conceitual, o conjunto de atores sociais presentes na rede analisada, as respectivas posições e recursos disponíveis, englobando-se as relações anteriormente existentes desses atores (MARQUES, 1998; 1999). Essa metodologia permite detalhar a interação dos atores sociais nos cenários sociopolíticos, com suas microrrelações e mudanças temporais. Nos processos decisórios e de gestão, estabelecidos entre 0 público e o privado, a análise das redes sociais "[...] permite a realização de investigações sofisticadas e diretas sobre os padrões de relação entre indivíduos e grupos, aproximando-nos dos tão decantados microfundamentos da ação social sem a perda de visão da estrutura." (MARQUES, 1999, p. 47).

As análises das conexões diretas e indiretas das relações na rede permitem identificar algumas tendências sociais que podem auxiliar na compreensão da proeminência desses atores e da potencial importância de cada um no enfrentamento à violência contra a mulher. A localização dos atores e seus atributos são elementos capazes de restringir a ação e/ou viabilizar oportunidades, o que demonstra a sua proeminência na rede (EMIRBAYER; GOODWIN, 1994; FREY; CZAJKOWSKI JR, 2005). 


\section{VIOLÊNCIA CONTRA A MULHER E O ESTABELECIMENTO DE UMA REDE DE ENFRENTAMENTO}

Localizado no Norte do Brasil, o Pará (Figura 1) possui 144 municípios divididos em 12 Regiões de Integração (RI), implantadas nos núcleos das cidades polo. Com uma população estimada em 8.513.497 habitantes, para o ano de 2014 (INSTITUTO BRASILEIRO DE GEOGRAFIA E ESTATÍSTICA, 2018), aproximadamente 46, 02\% residem na área rural (INSTITUTO BRASILEIRO DE GEOGRAFIA E ESTATÍSTICA, 2010).

O número de Delegacias Especializadas para as Mulheres (DEAMs) no Estado do Pará é 16. Em seis das DEAMs há o funcionamento das Unidades de Atendimento do PRO PAZ Integrado (PARÁ, [2015]). Conforme os dados levantados no portal da SPM, há 15 cidades com coordenadorias, secretarias ou superintendências instaladas. Mas em três Regiões de Integração: Tapajós, Xingu e Rio Capim ainda não há a presença da OPM. Nas informações do Departamento de Atividades Judiciais apresentados no Projeto Lenço e Documento do Ministério Público do Estado do Pará (PARÁ, [2015]), no interior do Estado há, aproximadamente, 40 mil registros relacionados à violência contra a mulher.

Figura 1 - Mapa do Estado do Pará - Regiões de Integração no Pará - Organismos Governamentais de políticas para as Mulheres

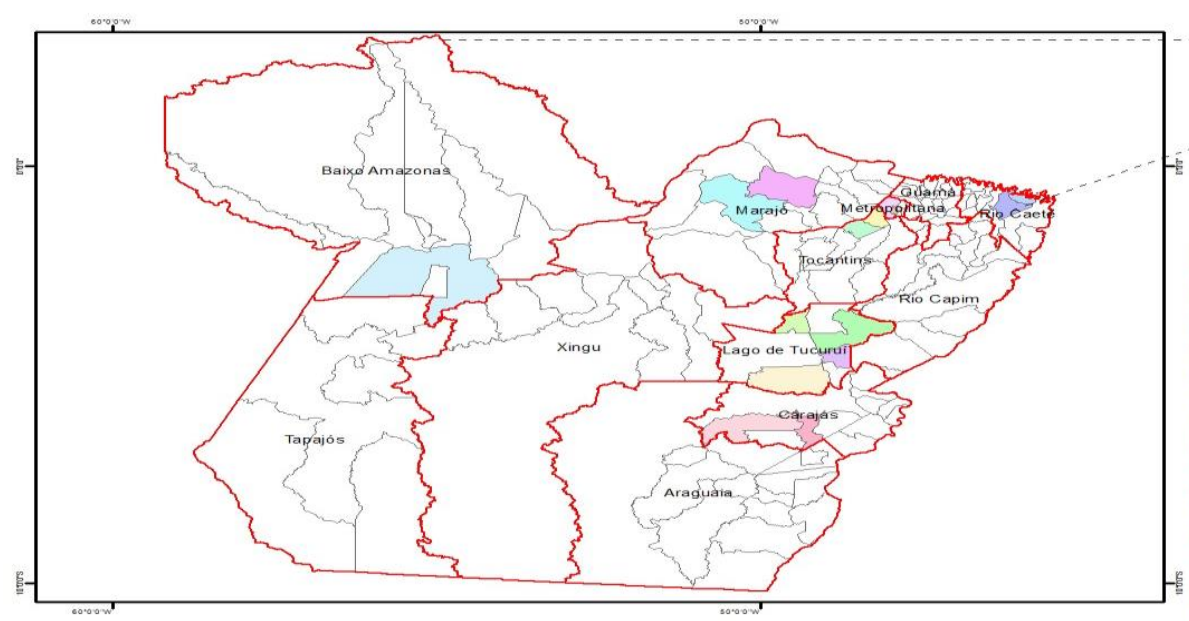

O 4,500 $9,000 \mathrm{Km}$

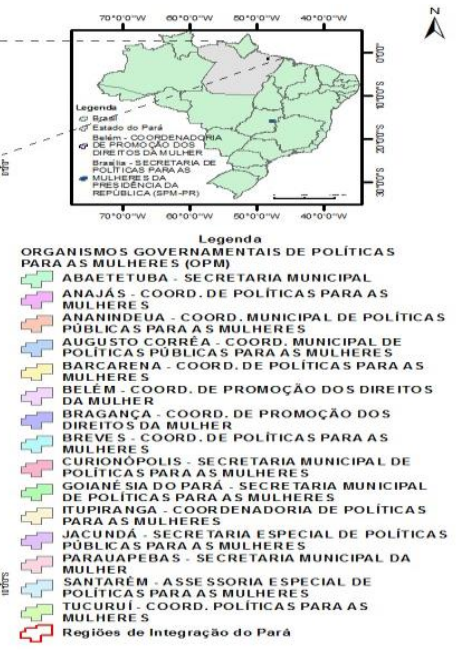

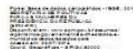

Fonte: BRASIL. Presidência da República. Secretaria de Políticas para as Mulheres. Guia para criação e implementação de organismos governamentais de políticas para as mulheres - OPM. Brasília: Secretaria de Políticas para as Mulheres, 2014. Disponível em: \&lt;http://www.spm.gov.br/central-de-conteudos/publicacoes/publicacoes/2014/guia-paraa-criacao-de-opm-dez2014.pdf\&gt;. Acesso em: 20 ago. 2015; INSTITUTO BRASILEIRO DE GEOGRAFIA E ESTATÍSTICA. Censo Demográfico. Rio de Janeiro, 2010. 
Uma das cidades que integra a lista dos maiores índices de violência abrangida pelo Programa PRO PAZ Integrado é Santarém, localizada na Região de Integração do Baixo Amazonas. A população estimada de Santarém, para o ano de 2018, é de 302.667 (INSTITUTO BRASILEIRO DE GEOGRAFIA E ESTATÍSTICA, 2019). No período de março de 2012 a fevereiro de 2014, houve 3258 atendimentos às mulheres vítimas de violência (CHAVES et al., 2014).

A Região de Integração do Marajó possui quase a totalidade das mulheres, da região 200 mil, vivendo na área rural, e encontram um acesso mais restrito à Justiça (PARÁ, 2015b). Baseado nos dados do Diagnóstico Socioeconômico e Ambiental da Região de Integração de Marajó (COSTA, 2018) é possível verificar que a região apresenta vulnerabilidade socioeconômica e educacional, fator que somado ao elemento geográfico se torna um desafio para alcançar as mulheres demandantes das políticas.

Uma das estratégias de ação em Marajó é a Rede de Atendimento às Mulheres em Situação de Violência, através da agência Barco PA - llha de Marajó. Outra ação é a oficina pela Cidadania das Mulheres Marajoaras, coordenada pela SNPM/PR em parceria com os atores públicos e privados do Estado (BRASIL, 2015). A construção de uma rede de enfrentamento e o compartilhamento de responsabilidades são imprescindíveis para a efetividade das ações propostas.

\section{RESULTADOS E DISCUSSÕES}

Como estamos lidando com muitas organizações, entidades e setores públicos, temos pouca capacidade de descrever apenas com a representação gráfica ou a descrição; logo, para entender essa rede de enfrentamento por meio da análise da rede social, serão verificados os cliques e as medidas de centralidade: centralidade de grau (degree), centralidade de proximidade (closeness); centralidade de intermediação (betweenness), centralidade de informação (information).

0 sociograma apresenta 0 seguinte desenho estrutural: a)- 0 grafo permitirá observar visualmente as relações e a posição dos atores; b)- Os membros do Fórum estão localizados no canto esquerdo. A parte superior da rede é composta pela Câmara, e, ao lado direito, têm-se os atores que participam do Conselho. Os quadrados azuis foram utilizados para demonstrar os atores políticos, e os círculos vermelhos os atores privados, formais e informais.

\section{Cliques}

A rede geral foi construída a partir dos três cliques: o Fórum, o Conselho e a Câmera Técnica. Como essas organizações são de caráter permanente, estabeleceu-se para fins de análise que os atores 
de cada uma dessas entidades estabelecem, no âmbito do seu espaço de atuação, relações diretas. Forma-se na análise da rede o que é caracterizado por cliques. Isso significa que cada uma das organizações é constituída por um conjunto de nós. Um nó corresponde a um ator. Dentro de um mesmo grupo, todos os nós se relacionam diretamente, configurando os cliques. (MARTELETO, 2001). A Rede Geral (Figura 2) permite visualizar as relações estabelecidas dentro do grupo e entre os três cliques. A parte central da rede é composta por atores que atuam de forma integrada nessa pauta de enfrentamento à violência contra as mulheres do campo, das florestas e das águas. Possivelmente, a centralidade ocorre porque todos os membros da Câmara Técnica são ligados ao Estado.

\section{Figura 2 - Rede Geral - Coordenadoria Integrada de Políticas para as Mulheres}

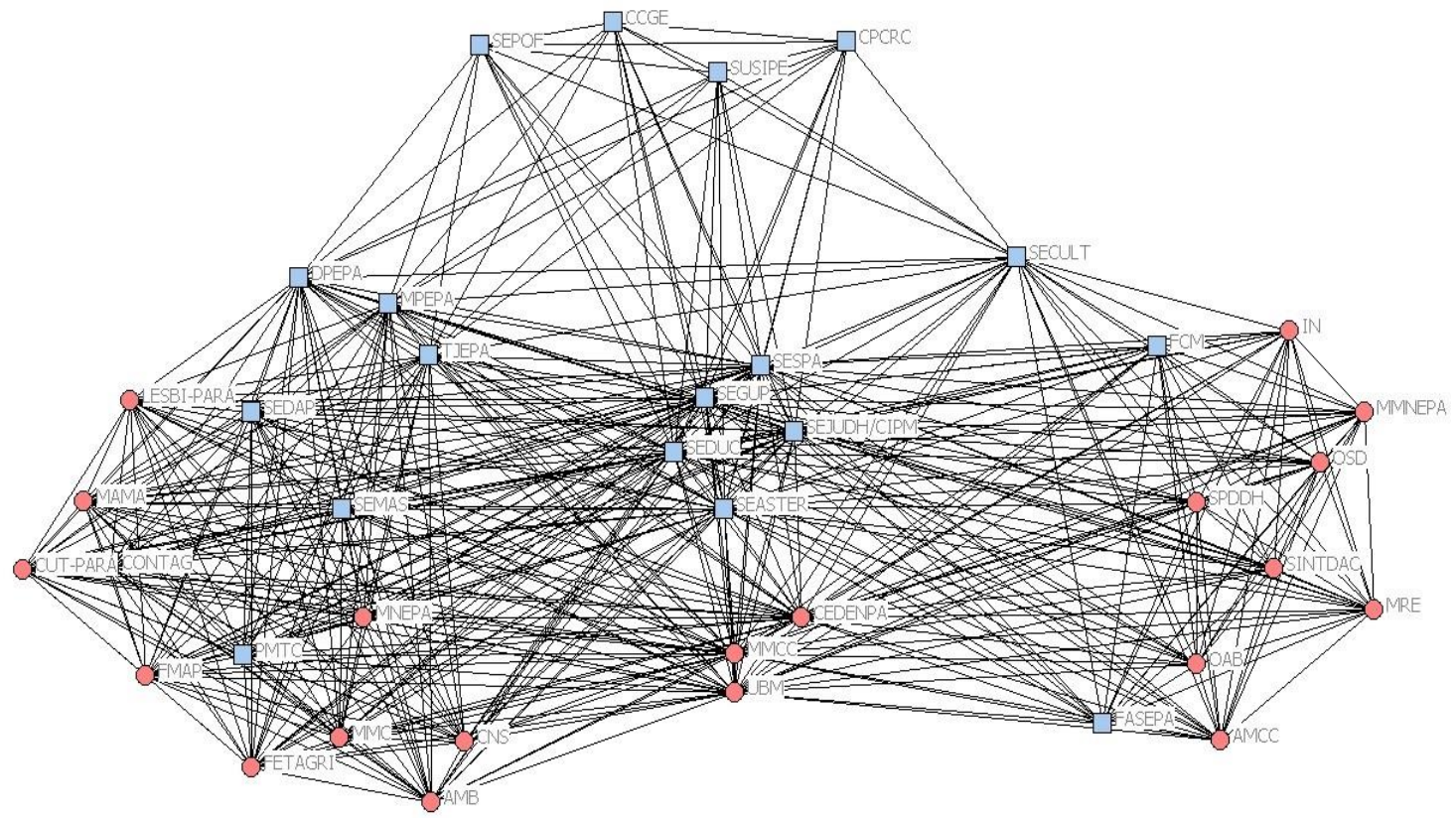

Fonte: Dados da pesquisa, 2015 - Levantamento de informações junto à Coordenadoria de Promoção de Políticas para as Mulheres do Estado do Pará, julho e agosto de 2015.

Os atores privados posicionados no centro inferior da rede cumprem um papel importante na ligação do Fórum e do Conselho, e aquelas estruturas nacionais repercutem a sua relevância na articulação com a esfera federal. Para além da representação gráfica é imprescindível realizar medições que sejam capazes de apontar a importância dos atores dentro da estrutura de rede. (MARTELETO, 2001; WASSERMAN; FAUST, 1999). 
Centralidade de informação

Se transmitida de forma eficiente, a capacidade de trocar informações importantes para 0 propósito da rede pode implicar maior liderança entre os pares (Tabelas 1 e 2). (TOMAÉL; MARTELETO, 2006).

\section{Tabela 1 - Centralidade de Informação}

\begin{tabular}{l|r}
\hline CEDENPA & 13,47 \\
\hline DPEPA & 12,37 \\
\hline MMCC & 13,47 \\
\hline MPEPA & 12,37 \\
\hline SEASTER & 14,09 \\
\hline SEDUC & 14,09 \\
\hline SEGUP & 14,09 \\
\hline SEJUDH/CIPM & 14,09 \\
\hline SESPA & 14,09 \\
\hline TJEPA & 12,37 \\
\hline UBM & 13,47 \\
\hline
\end{tabular}

Fonte: Dados da pesquisa, 2015 - Levantamento de informações junto à Coordenadoria de Promoção de Políticas para as Mulheres do Estado do Pará, julho e agosto de 2015.

\section{Tabela 2 - Centralidade de Grau e Número de Contatos}

\begin{tabular}{l|c|c}
\hline CEDENPA & 34 & 89,47 \\
\hline DPEPA & 28 & 73,68 \\
\hline MMCC & 34 & 89,47 \\
\hline MPEPA & 28 & 73,68 \\
\hline SEASTER & 38 & 100 \\
\hline SEDUC & 25 & 100 \\
\hline SEGUP & 38 & 100 \\
\hline SEJUDH/CIPM & 38 & 100 \\
\hline SESPA & 38 & 100 \\
\hline TJEPA & 28 & 89,47 \\
\hline UBM & 34 & 89,47 \\
\hline Fo:
\end{tabular}

Fonte: Dados da pesquisa, 2015 - Levantamento de informações junto à Coordenadoria de Promoção de Políticas para as Mulheres do Estado do Pará, julho e agosto de 2015.

Nessas medidas de centralidade, observa-se o poder de informação que os movimentos para as mulheres do campo possuem: União de Mulheres Brasileiras (UBM), Movimento Mulheres do Campo e das Cidades (MMCC), Centro de Defesa do Negro do Pará (CEDENPA). Esses movimentos e a Contag, que obtiveram medida de informação de 11,25 , são relevantes para o estabelecimento de pautas sobre a 
violência contra as mulheres do campo e das florestas, não apenas no Pará, mas no Brasil. Esse tipo de centralidade dá para os atores uma capacidade de colocar a agenda e pautar temas devido à posição que eles ocupam na rede.

O índice de informação do Tribunal de Justiça do Estado do Pará (TJEPA) e do Ministério Público do Estado do Pará (MPEPA) demonstra a importância desses dois atores públicos na rede. Inseridos diretamente na rede de enfrentamento e de atendimento à violência doméstica e familiar, figuram como centrais para o sucesso da ação, pois são órgãos que dependem do acesso de informação para garantir um resultado efetivo nesse tipo de ação.

Centralidade de grau

Destaca-se a centralidade do grau para a coordenação dos projetos a serem desenvolvidos pela CIPM. Como esses atores são os que detêm mais laços nas redes, são eles que estabelecem de forma sistemática o contato com os mais diferentes atores.

Quatro secretarias e a Coordenadoria concentraram a centralidade do grau na rede: SEJUDH/CIPM, Secretaria de Estado de Segurança Pública e Defesa Social (SEGUP), Secretaria de Estado de Educação (SEDUC), Secretaria de Estado de Assistência Social, Trabalho, Emprego e Renda (SEASTER) e Secretaria de Estado de Saúde Pública (SESPA). Todos esses atores têm posições estruturais capazes de gerenciar de forma direta a rede das ações de enfrentamento à violência, pois todas apresentam 38 contatos estabelecidos.

A participação desses atores na Câmara Técnica, no Fórum, no Conselho é o que faz com que tenham a possibilidade de exercer um papel protagonista na política. A própria competência administrativa da Coordenadoria e das secretarias também implica uma maior centralidade na rede de combate à violência contra a mulher no Estado do Pará.

Entretanto, não há uma obrigatoriedade de maior articulação ou proximidade em razão do número de laços diretos que o nó estabelece. (MARTELETO, 2001).

A capacidade de articulação e movimento por diversos atores pode ser compreendida de forma sistemática a partir das outras duas centralidades na rede geral: proximidade e intermediação. 
Centralidade de intermediação

0 grau de intermediação (betweeness) se refere à capacidade de estabelecer sub-redes e estabelecer intermediações entre os grupos. Os atores são capazes de estabelecerem filtros e concentrarem influência e poder na rede. (MARTELETO, 2001).

$\mathrm{Na}$ Tabela 3 podem ser destacadas as principais organizações que mediam esses recursos de intermediação. Os cinco atores que apresentaram o maior grau de centralidade de grau apresentam 0 papel protagonista na intermediação. É importante destacar o papel dos outros atores em proeminência nesta medida.

\section{Tabela 3 - Centralidade de Intermediação}

\begin{tabular}{c|c}
\hline DPEPA & 1282 \\
\hline MMCC & 2954 \\
\hline MPEPA & 1282 \\
\hline SEASTER & 5016 \\
\hline SEDUC & 5016 \\
\hline SEGUP & 5016 \\
\hline SEJUDH/CIPM & 5016 \\
\hline SESPA & 5016 \\
\hline TJEPA & 1,282 \\
\hline UBM & 2,954 \\
\hline SECULT & 1,62 \\
\hline CEDENPA & 2954 \\
\hline
\end{tabular}

Fonte: Dados da pesquisa, 2015 - Levantamento de informações junto à Coordenadoria de Promoção de Políticas para as Mulheres do Estado do Pará, julho e agosto de 2015.

Dois atores apresentam um papel relevante de intermediação: o Ministério Público do Estado Pará (MPEPA) e o Tribunal de Justiça do Estado do Pará (TJEPA). Ambos apresentaram um índice de intermediação de 1.28. É interessante destacar como essa centralidade, nesse caso, coincide com aquela provocada por informação, exatamente pela aproximação de distintas sub-redes e grupos.

Os dois órgãos e o Estado do Pará assinaram no dia 18 de agosto de 2015 um acordo de cooperação técnica para que sejam efetivadas medidas de ações para fortalecer a política estadual de enfrentamento à violência contra as mulheres. (BRASIL, 2015).

No TJEPA, a ação de enfrentamento à violência ocorre por meio da Coordenadoria Estadual das Mulheres em Situação de Violência Doméstica e Familiar. Com a atuação de juízes e servidores no 
TJEPA, na DEAM, integrado ao PRO PAZ, como publicado no Diário do Pará, dia $1^{\circ}$ de setembro de 2015 (PARÁ, 2015a).

No Núcleo de Enfrentamento à Violência contra a Mulher do MPEPA os órgãos que compõem a rede de proteção são: o Judiciário, por meio da Vara do Juizado de Violência Doméstica Familiar contra a mulher; Promotoria de Violência Doméstica, Promotoria Especializada do Ministério Público; DEAM, Delegacia Especializada de Atendimento à Mulher (integra a estrutura da Polícia Civil e atua na esfera da Violência Doméstica); Defensoria Pública, Núcleo de Atendimento Especializado à Mulher Vítima de Violência Doméstica; Abrigos; Equipe Multidisciplinar; IML - Centro de Perícias; PRO PAZ-Mulher; SPM Secretaria de Políticas para Mulheres da Presidência da República (PARÁ, [2015]).

Os movimentos como a UBM, o MMCC e o CEDENPA formam o segundo grupo, que apresentou o maior índice de intermediação. Isso mostra a potencial relevância da sociedade civil organizada na ação de enfrentamento à violência no Estado do Pará. Os três são estratégicos por serem atores porteiros com outros movimentos, não apenas direcionados à população negra, mas aos movimentos de mulheres e aos diversos grupos étnicos.

A possibilidade de estabelecer pontes além do limite da rede do CIPM impacta na própria centralidade da CIPM e nas demais relações com atores que atuam na rede de enfrentamento e de atendimento.

Centralidade de proximidade

Essa centralidade demonstra quais as organizações que possuem uma "[...] independência em relação ao controle dos outros." (MARTELETO, 2001, p. 78). À medida que determinados atores realizam o contato de forma independente com as organizações-chaves para a rede de enfrentamento à violência, eles poderão ser utilizados de forma estratégica nas articulações institucionais.

Nessa medida de centralidade há movimentos sociais que se destacam, embora não tenham apresentado índice de intermediação: Articulação de Mulheres Brasileiras (AMB), Movimento das Mulheres Camponesas (MMC) e o Movimento Articulado de Mulheres da Amazônia (MAMA). (Tabela 4) 
Tabela 4 - Centralidade de Proximidade

\begin{tabular}{l|c}
\hline DPEPA & 79.16 \\
\hline MMCC & 90.47 \\
\hline MPEPA & 79.16 \\
\hline SEASTER & 100.00 \\
\hline SEDUC & 100.00 \\
\hline SEGUP & 100.00 \\
\hline SEJUDH/CIPM & 100.00 \\
\hline SESPA & 100.00 \\
\hline TJEPA & 79.16 \\
\hline UBM & 90.47 \\
\hline SECULT & 74.51 \\
\hline CONTAG & 71.69 \\
\hline LESBIIPARA & 71.69 \\
\hline MMC & 71.69 \\
\hline MAMA & 71.69 \\
\hline CEDENPA & 90.47 \\
\hline FoI
\end{tabular}

Fonte: Dados da pesquisa, 2015 - Levantamento de informações junto à Coordenadoria de Promoção de Políticas para as Mulheres do Estado do Pará, julho e agosto de 2015.

Criada há 11 anos, a AMB. se constitui como uma organização política feminista que tem, entre seus propósitos promover o fortalecimento desses movimentos em âmbito local e regional (ARTICULAÇÃO DE MULHERES BRASILEIRAS, 2015). Por essa razão, há a possibilidade de a AMB ser uma importante ponte com os outros atores públicos e privados que não estão inseridos na Rede Geral, podendo, dessa forma, potencializar a sua atuação em regiões não contempladas pela Coordenadoria.

$O$ caráter organizativo do MMC com base nas direções estaduais, regionais ou nacionais também poderá fortalecer as ações nas regiões rurais do Estado do Pará. $O$ enfrentamento coletivo, por meio da integração entre as bases comunitárias e as organizações, o estímulo às práticas cotidianas de conscientização e de uma cultura de igualdade entre homens e mulheres, as denúncias das diversas tipificações de violência e a elaboração de políticas públicas direcionadas a igualdade de gênero são os maiores desafios que o MCC encontra nas suas atividades. (MOVIMENTO MULHERES CAMPONESAS, 2019).

Verifica-se que o MAMA, embora não tenha apresentado índice de intermediação, possui a capacidade de estabelecer vínculos com outros nós da rede que estejam aparentemente distantes de suas relações. A proximidade pode colaborar para a elaboração de estratégias e de fluxo de informações que serão importantes nos projetos de combate à violência contra as mulheres indígenas. Esse grupo étnico 


\section{ARTICULAÇÃO E REDES NO ENFRENTAMENTO À VIOLÊNCIA CONTRA AS MULHERES NO PARÁ}

feminino permanece, ainda, sem a abrangência necessária ou adequada à realidade das comunidades indígenas na elaboração das políticas públicas. (CASTILHO, 2008; TORRES, 2010; VERDUM, 2008).

A Contag possui a capacidade de alcançar outros atores distantes na rede de forma rápida e independente. Além disso, a Contag possui uma importância no contexto dos movimentos para as mulheres rurais na esfera nacional. Por meio da coordenação das Marchas das Margaridas, e das suas relações com os diversos movimentos sociais e os grupos feministas, apresenta um papel relevante para 0 fortalecimento das redes de enfrentamento à violência e como propositora de pautas, como pode ser verificado nos próprios textos de formação da SPM/PR. (BRASIL 2011a, 2011b).

A Casa Civil da Governadoria do Estado (CCGE), a Superintendência do Sistema Penitenciário (SUSIPE), Secretaria de Estado de Planejamento, Orçamento e Finanças (SEPOF) e o Centro de Perícias Científicas Renato Chaves, embora componentes da Câmara Técnica de Monitoramento do Pacto Nacional de enfretamento a Violência Contra a Mulher, nesse recorte estabelecido, apresentaram os menores graus de proximidade e nenhum grau de intermediação (Quadro 3).

\section{CONCLUSÃO}

É importante ressaltar que a verificação da posição de cada um desses atores na estrutura da rede e sua proeminência (MARTELETO, 2001; FREY; CZAJKOWSKI JR, 2005) pode ser importante para a reavaliação da ação e da importância de cada ator nas estratégias de enfrentamento à situação de violência da mulher.

Entretanto, é necessária uma análise detalhada da atuação de cada ator e de cada subgrupo na rede para verificar se no âmbito da sua competência há a efetividade nas ações de enfrentamento à violência.

Os resultados dos dados quantitativos demonstraram que os principais atores responsáveis pelas articulações de combate à violência são as secretarias de Estado. A elas cabe o principal papel de articular as políticas e de intermediar os atores da rede. É importante, portanto, refletir que a integração dos subgrupos e dos atores é primordial para que as políticas sejam implementadas estrategicamente.

A capacidade de informação e de estabelecimento de contatos desses movimentos e organizações não-governamentais dentro da rede pode potencializá-la para ampliar seus laços com as sub-redes em lugares de difícil acesso do Pará. E, dessa forma, criar estratégias para alcançá-los por meio dos seus próprios atores locais. É importante também destacar o papel que essas organizações da 
sociedade civil podem cumprir na construção de agendas e pautas, apesar de não ter centralidades relacionadas à coordenação e articulação. O Núcleo de Enfrentamento à Violência contra a Mulher do Ministério Público do Pará apresentou nos dados da rede o que o levantamento qualitativo já havia indicado: um importante papel no enfrentamento à violência.

Supõe-se que o fortalecimento e a criação dos organismos municipais de políticas para as mulheres poderá ser uma das estratégias de operacionalização das políticas de enfrentamento à violência contra a mulher em regiões de difícil acesso.

Uma das dificuldades encontradas para desenvolver este estudo foi o acesso a um sistema dados atualizados sobre o mapeamento da violência contra as mulheres nessas populações específicas. Como já apontado no documento Mulheres das Matas e das Florestas: Diretrizes e Ações de Enfretamento (BRASIL, 2011b), essas informações integradas poderão servir de base para o planejamento estratégico da rede de enfrentamento.

As mulheres do Marajó, entre tantas outras, aguardam que a formação da rede de enfrentamento se fortaleça e as alcance nas terras amazônicas paraenses.

\section{REFERÊNCIAS}

ARTICULAÇÃO DE MULHERES BRASILEIRAS. Site. [S. I.], 2015. Disponível em: http://www.articulacaodemulheres.org.brl. Acesso em: 20 jun. 2019.

\section{BARROSO, M. F. Rotas Críticas das Mulheres Sateré-Mawé no enfrentamento da violência} doméstica: novos marcadores de gênero no contexto indígena. 2011. Dissertação (Mestrado em Serviço Social) - Programa de Pós-graduação em Serviço Social, Universidade Federal da Amazônia, Manaus, 2011.

BARSTED, L. L. A violência contra as mulheres no Brasil e a Convenção de Belém do Pará dez anos depois. In BARSTED, L. L.; PITANGUY, J.; MIRANDA, D. (orgs.) O Progresso das Mulheres no Brasil. Rio de Janeiro: Cepia, 2006a. p. 246-289.

BARSTED, L. L. O avanço legislativo no enfrentamento da violência contra as mulheres. In LEOCÁDIO, E.; LIBARDONI, M. (org.). 0 desafio de construir redes de atenção às mulheres em situação de violência. Brasilia: Agende, 2006b. p. 65-89.

BARSTED, L. L. O progresso das mulheres no enfrentamento da violência. In BASTERD, L. L.; PITANGUY, J. (org). O Progresso das Mulheres no Brasil 2003- 2010. Rio de Janeiro: CEPIA; Brasília: ONU Mulheres, 2011. p. 346-382.

BRASIL. Ministério da Mulher, da Família e dos Direitos humanos. Secretaria de Políticas para as Mulheres. Comunicação Social. SPM promove oficina pela Cidadania das Mulheres Marajoaras. 
Brasília, DF, 2015. Disponível em: https://www.mdh.gov.br/noticias-spm/noticias/spm-promove-oficina-pelacidadania-das-mulheres-marajoaras. Acesso em: 23 jun. 2019.

BRASIL. Presidência da República. Secretaria de Políticas para as Mulheres. Guia para criação e implementação de organismos governamentais de políticas para as mulheres - OPM. Brasília: Secretaria de Políticas para as Mulheres, 2014. Disponível em: \&lt;http://www.spm.gov.br/central-deconteudos/publicacoes/publicacoes/2014/guia-para-a-criacao-de-opm-dez2014.pdf\&gt;. Acesso em: 20 ago. 2015.

BRASIL. Presidência da República. Secretaria Nacional de Enfrentamento à Violência contra as Mulheres. Secretaria de Políticas para as Mulheres. Rede de Enfrentamento à Violência contra as Mulheres. Brasília, DF, 2011a. Disponível em: http://www.spm.gov.br/sobre/publicacoes/publicacoes/2011/rede-deenfrentamento. Acesso: 15 jun. 2015.

BRASIL. Presidência da República. Secretaria Nacional de Enfrentamento à Violência contra as Mulheres. Secretaria de Políticas para as Mulheres. Mulheres do Campo e da Floresta: Diretrizes e ações nacionais. Brasília, DF, 2011d Disponível em:

http://www.spm.gov.br/sobre/publicacoes/publicacoes/2011/campo-e-florestaOrgao=81. Acesso: 15 jun. 2015.

CASTILHO, E. W. A violência doméstica contra a mulher no âmbito dos povos indígenas: qual lei aplicar? In VERDUM, R. (org.). Mulheres Indígenas, Direitos e Políticas Públicas. Brasília, DF: INESC, 2008. p. 21-32.

CHAVES, J. F. et al. A tecnologia para a garantia de direitos: o Sistema de Informação do PRO PAZ Integrado para a garantia dos direitos humanos a partir das práticas de controle e prevenção das violências. In ENCONTRO NACIONAL DA ANDHEP: Políticas Públicas para a Segurança Pública e Direitos Humanos, 8., 2014, São Paulo. Anais... São Paulo: ANDHEP, 2014. p. 1-24. Disponível em: http://www.encontro2014.andhep.org.br/resources/anais/1/1397478618_ARQUIVO_(ARTIGO-ANDHEPGT15-Atecnologiaparagarantiasdedireitos.pdf. Acesso em: 22 ago. 2015.

COSTA, E. Diagnóstico Socioeconômico e Ambiental da Região de Integração do Marajó. Belém: FAPESPA, 2018. Disponível em: http://feiradoempreendedorpa.com.br/site/downloads/inteligenciademercado/oportunidadedenegocios/058.p df. Acesso em: 30 nov 2018.

DARON, V. Um Grito Lilás: cartografia da violência às mulheres do campo e da floresta. Brasília, DF: Secretaria de Políticas para as Mulheres, 2009. Disponível em: http://www.mulheres.gov.br/arquivosdiversos/sev/coordenacao-geral-de-acoes-preventivas-e-garantia-de-direitos/enfrentamento-a-violenciacontra-as-mulheres-do-campo-e-da-floresta/documentos/um\%20grito\%20lilas.pdf. Acesso em: 15 jul 2015.

DURÁN, F. E. Viejas e nuevas imágenes sociales de ruralidad. Estudos Sociedade e Agricultura, Rio de Janeiro, n. 11, p. 76-98, 1998. Disponível em: http://www.revistaesa.com/ojs/index.php/esa/article/view/136. Acesso em: 12 maio 2019. 
EMIRBAYER, M.; GOODWIN, J. Network analysis, culture and problem of agency. American Journal of Sociology, Chicago, v. 99, n. 6, p. 1411-1454, 1994.

FREY, K.; CZAJKOWSKI JR, S. O município e a segurança pública: o potencial da governança democrática urbana. Revista de Administração Pública, Rio de Janeiro, v. 39, n. 2, p. 297-325, 2005b.

INSTITUTO BRASILEIRO DE GEOGRAFIA E ESTATÍSTICA. Cidades e Estados: Santarém. Rio de Janeiro, 2019. Disponível em: https://www.ibge.gov.br/cidades-e-estados/pa/santarem.html. Acesso em: 23 jun. 2019.

INSTITUTO BRASILEIRO DE GEOGRAFIA E ESTATÍSTICA. Censo Agropecuário. Rio de Janeiro, 2017. Disponivel em: https://www.ibge.gov.br/estatisticas-novoportal/economicas/agricultura-e-pecuaria/218142017-censo-agropecuario.html?=\&t=resultados. Acesso em: 24 out 2018.

INSTITUTO BRASILEIRO DE GEOGRAFIA E ESTATÍSTICA. Censo Demográfico. Rio de Janeiro, 2010.

INSTITUTO BRASILEIRO DE GEOGRAFIA E ESTATÍSTICA. Panaroma Cidades: Santarém. Rio de Janeiro, 2018a. Disponivel em: https://cidades.ibge.gov.br/brasil/pa/santarem/panorama. Acesso em: 30 nov 2018.

MARQUES, E. C. L. Redes sociais e instituições na construção do Estado e sua permeabilidade. Revista Brasileira de Ciências Sociais, São Paulo, v. 14, n. 41, p. 45-67, 1999. Disponível em: http://www.scielo.br/pdf/\%0D/rbcsoc/v14n41/1751.pdf. Acesso em: 12 maio 2019.

MARQUES, E. C. L. Redes sociais e permeabilidade do Estado: instituições e atores políticos na produção da infra-estrutura urbana no Rio de Janeiro. 1998. Tese (Doutorado em Ciências Sociais). Instituto de Filosofia e Ciência Humanas da Universidade Estadual de Campinas, 1998.

MARTELETO, R. M. Análise de redes sociais: aplicação nos estudos de transferência da informação. Ciência da Informação, Brasília, DF, v. 30, n. 1, p. 71-81, 2001. Disponível em: http://www.scielo.br/pdf/ci/v30n1/a09v30n1.pdf. Acesso em: 15 jul. 2015.

MATOS, E. A. C. de; MEDEIROS, R. M. V. A Relação Campo-Cidade e as Novas Ruralidades. ParaOnde!?, Porto Alegre, v. 5, n. 1, p. 1-15, 2011.

MEDEIROS, R. M. V. Ruralidades: novos significados para o tradicional rural. In MEDEIROS, R. M. V.,LINDNER, M. (orgs.). Dinâmicas do espaço agrário: velhos e novos territórios : NEAG: 10 anos. Porto Alegre: Evangraf, 2017. p. 179-189. Disponivel em: http://www.lume.ufrgs.br/handle/10183/157495. Acesso em: 9 maio 2019.

MOVIMENTO MULHERES CAMPONESAS. História. [S. I.], 2019. Disponível em: http://www.mmcbrasil.com.br/site/. Acesso em: 20 jun. 2019.

PARÁ. Ministério Público do Pará. Rede de Proteção. Belém, [2015]. Disponível em: https://www2.mppa.mp.br/gcsubsites/index.php?action=Orgao.site\&oOrgao=81. Acesso em: 30 ago. 2015. 
PARÁ. Tribunal de Justiça do Estado do Pará Atendimento à mulher é regulamentado. Portaria designa magistrados para atuarem no Pro Paz Mulher. Belém, 2015a. Texto de Will Montenegro. Disponível em: http://www.tjpa.jus.br/PortalExterno/imprensa/noticias/Informes/26687-Judiciario-regulamenta-atendimentono-ProPaz-Mulher.xhtml Acesso em: 2 set. 2015.

PARÁ. Tribunal de Justiça do Estado do Pará. Coordenadoria de Imprensa. Mulheres do Marajó debatem violência. Belém, 2015b. Texto de João Vital. Disponível em:

http://www.tjpa.jus.br/PortalExterno/imprensa/noticias/Informes/2645-Mulheres-do-Marajo-debatemviolencia.xhtml. Acesso: 25 jul. 2015.

SUÁREZ R, N. del C. Procesos y dinámica rurales: una lectura desde el enfoque de género. Revista Luna Azul, Manizales, n. 27, p. 94-103, 2008. Disponível em:

http://www.redalyc.org/pdf/3217/321727229008.pdf. Acesso em: 23 jun. 2019.

SUZIKI, J. C. Campo e cidade no Brasil: transformações socioespaciais e dificuldades de conceituação.

Revista NERA, Presidente Prudente, v. 10, p. 134-150, 2007. Disponível em:

http://mstemdados.org/sites/default/files/1429-4137-1-PB.pdf. Acesso em: 15 ago 2015.

TOMAÉL, M. I.; MARTELETO, R. M. Redes sociais: posições dos atores no fluxo da informação.

Encontros Bibli: Revista Eletrônica de Biblioteconomia e Ciência da Informação, Florianópolis, v. esp., p. 75-91, 2006. Disponível em: https://periodicos.ufsc.br/index.php/eb/article/view/15182924.2006v11nesp1p75. Acesso em: 15 ago. 2015.

TORRES, M. S. Um olhar sobre a violência intrafamiliar em Aldeias Karajá. In: FAZENDO GÊNERO: Diásporas, Diversidades, Deslocamentos, 9., 2010, Curitiba. Anais... Curitiba: Universidade Federal de Santa Catarina, 2010. Disponível em:

http://www.fazendogenero.ufsc.br/9/resources/anais/1266957895_ARQUIVO_Umolharsobreaviolenciaintraf amiliaremaldeiasKaraja.pdf. Acesso em: 20 jun. 2019.

VERDUM, R. Mulheres indígenas, direitos e políticas públicas. In VERDUM, R. (org). Mulheres Indígenas, Direitos e Políticas Públicas. Brasília, DF: INESC, 2008. p. 7-20.

WASSERMAN, S.; FAUST, K. Social Network Analysis: methods and Applications. Cambridge, UK: Cambridge University Press, 1999. 